\title{
Transport of angular momentum in solar-like oscillating stars
}

\author{
Mariejo Goupil ${ }^{1}$, Sébastien Deheuvels ${ }^{2}$, Joao Marques ${ }^{3}$, \\ Yveline Lebreton $^{4,5}$, Benoit Mosser ${ }^{1}$, Rafa García ${ }^{6}$, Kevin Belkacem ${ }^{1}$, \\ and Stéphane Mathis ${ }^{6}$ \\ ${ }^{1}$ Observatoire de Paris, LESIA, CNRS UMR 8109, F-92190 Meudon, France \\ email: mariejo.goupil@obspm.fr \\ ${ }^{2}$ Université de Toulouse, UPS-OMP IRAP, CNRS, F-31400 Toulouse, France; \\ ${ }^{3}$ Institut d'Astrophysique Spatiale, UMR 8617, CNRS, Université Paris XI, \\ 91405 Orsay Cedex, France; \\ ${ }^{4}$ Observatoire de Paris, GEPI, CNRS UMR 8109, F-92190 Meudon, France; \\ ${ }^{5}$ Institut de Physique de Rennes, Université de Rennes 1, CNRS UMR 6251, \\ F-35042 Rennes, France; \\ ${ }^{6}$ Laboratoire AIM Paris-Saclay, CEA/DSM-CNRS-Université Paris Diderot, IRFU/SAp \\ Centre de Saclay, 91191 Gif-sur-Yvette, France
}

\begin{abstract}
Our current understanding and modeling of angular momentum transport in lowmass stars are briefly reviewed. Emphasis is set on single stars slightly younger that the Sun and on subgiants and red giants observed by the space missions CoRoT and Kepler.
\end{abstract}

Keywords. stars: solar-type, stars: oscillations, stars: rotation, stars: evolution, stars: interiors

\section{Introduction and background}

All stars rotate to some extent from their birth throughout their lifetimes. The evolution of angular momentum (AM) therefore is an essential feature of stellar evolution. For a star rotating as a solid body, the total specific AM is $j=k^{2} R^{2} \Omega$, where $\Omega$ is the rotation rate, $R$ is the stellar radius and $k$ is a constant of order unity. When no external torque is applied, the AM of the system is conserved and one must expect an increase of $\Omega$ when the star contracts - during the pre-main sequence (PMS) for instance - and a slowing down of expanding stellar regions - such as during the subgiant and red giant phase. Observations however indicate that low-mass stars slow down during most of their lifetime, and develop differential rotation in radius. Contracting regions that are expected to rotate quite fast do not, while expanding regions which ought to rotate slowly rotate faster than expected. This means that AM transport occurs within stars. The issue then is to identify and model the braking processes that cause the internal transport and evolution of stellar AM of low -mass stars. This review is limited to 'isolated' stars (no binaries or effects from planets), and focuses on low-mass stars of $\sim 0.9-1.5 M_{\odot}$.

The convective regions of one-dimensional stellar models are assumed instantaneously chemically homogenized and in solid-body rotation (but see Potter 2012 and Brun \& Palacios 2009). In radiative regions, one needs to solve a time-dependent equation for the local specific AM, $j(r)=r^{2} \Omega(r)$ of the form:

$$
\frac{\partial j}{\partial t}+\dot{r} \frac{\partial j}{\partial r}=-\frac{1}{\rho r^{2}} \frac{\partial\left(r^{2} \mathcal{F}\right)}{\partial r}+\left(\frac{d j}{d t}\right)_{\mathrm{ext}},
$$

where $r(m)$ is the radius enclosing the mass $m, \dot{r}$ the time derivative of the radius and $\mathcal{F}(r)$ is the angular momentum flux. In order to solve Eq. (1.1), one must specify $\mathcal{F}(r)$, 
the surface AM losses $(d j / d t)_{\text {ext }}$ and the initial condition $j_{0}(r)=j(r, t=0)$. The AM flux is the result of several AM transport processes (see Zahn 2007, Talon 2008a, Maeder 2009, and Mathis 2013 for reviews). It is given by $\mathcal{F}(r)=\mathcal{F}_{\mathrm{MC}}(r)+\mathcal{F}_{\text {turb }}(r)+\mathcal{F}_{\mathrm{IGW}}(r)+\mathcal{F}_{\mathrm{B}}(r)$ where the currently identified transport processes are:

- Turbulent transport: Turbulence in radiative regions is generated by instabilities of various types (Endal \& Sofia 1978, Talon 2008a). Their combined effect is modeled as a diffusive process and contributes to a total turbulent viscosity $\nu_{v}$. The AM flux then is $\mathcal{F}_{\text {turb }}=-\rho r^{2} \nu_{v}(\partial \Omega / \partial r)$. This process transports AM from inner fast rotating layers to outer slower regions and can be dominant in regions with sharp rotation gradients. Several evolutionary codes include the AM transport only as a turbulent diffusion description (see for instance Denissenkov et al. 2010, Paxton 2013). The diffusion coefficients for the $\mathrm{AM}$ and chemical elements are then calibrated by fitting appropriate observations such as those for the Sun or open clusters. The validity of these calibrated values cannot be general. Besides, turbulent transport as a diffusion process cannot account for all possible types of AM transport.

- Transport by meridional circulation: Large-scale motions are driven by internal stresses, surface AM losses and structural changes. Zahn (1992) proposed to describe the combined effect of advective transport by meridional circulation and the diffusive (differential rotation) shear-induced turbulence as a 1D process (here CMST approach). The basic assumption is that a shear instability, acting on dynamical timescales leads to a large horizontal turbulent viscosity. This causes rapid homogenization on horizontal surfaces, and the rotation rate is a function of radius only (shellular rotation). The meridional circulation $\mathrm{AM}$ flux then is $\mathcal{F}_{\mathrm{MC}}=-\rho r^{2} \Omega U_{r} / 5$, where $U_{r}$ is the vertical meridional circulation velocity. Zahn (1992), Maeder \& Zahn (1998), and Mathis \& Zahn (2004) derived prescriptions for the transport coefficients for the coupled evolutions of AM and chemical elements (see Mathis 2013 for a recent review). Several codes have implemented this approach (Palacios 2013). It does however suffer from several uncertainties in the prescriptions of the transport coefficients (Meynet 2013, Maeder et al. 2013).

- Transport by internal gravity waves (IGW) in stellar radiative regions was first discussed by Press (1981) and Schatzman (1993). IGW transport has been proven to be efficient to transport AM and influence the chemical mixing. It is able to make the solar rotation rigid and to reproduce the cool side of the Li dip. The AM flux in that case results in successive AM-extraction propagating fronts from the inner to the outer layers. See reviews such as Talon (2008a) and Palacios (2013). Several open issues on the wave generation and propagation still cast some uncertainties on the quantitative efficiency of this transport for determining the rotation profile in radiative regions (Lecoanet \& Quataert 2013, Alvan et al. 2013; see also Mathis 2013 and references therein).

- Fossil magnetic fields and hydromagnetic instabilities. A fossil magnetic field is able to make the rotation nearly uniform (Mestel 1953). Its dynamics and its interactions with meridional currents, differential rotation and turbulence are complex (Mathis 2011 and references therein). The result as to whether such a process is able to enforce a rigid rotation in the solar radiative region, for instance, is still debated (Zahn 2009, Strugarek et al. 2011, Garaud et al. 2013).

- A magnetized wind has long been identified as an important process responsible for AM surface losses (Schatzman 1962, Skumanich 1972). Its description led to a prescription for $(d j / d t)_{\text {ext }}$ to be used in stellar models (Kawaler 1988) which involves a proportionality constant, $K$. The $K$ value is usually set by imposing the rotation period of the present Sun at the age of the Sun. However a realistic description of magnetized wind-driven AM losses remains quite complex and theoretical work is still ongoing to improve the prescription for $(d j / d t)_{\text {ext }}$ (Reiners \& Mohanty 2012, Matt et al. 2012). 
- The AM evolution must be coupled to that of the chemical elements. The evolution of the chemical elements is described as a diffusive process with a transport coefficient including impact of the meridional circulation, turbulence, etc. (Zahn 1992, Maeder \& Zahn 1998, Pinsonneault 1997, Talon 2008a, Palacios 2013).

Studies of the interactions of these different processes in terms of rotation profiles and mixing processes benefit nowadays from the results of several 3D numerical simulations (for instance Brun \& Rempel 2009, Rogers et al. 2006).

\section{Evolution of stellar AM}

The evolution of AM in stellar interiors of low-mass stars from the PMS up to advanced stages has been the subject of many papers, reviews and lectures, for instance Talon (2008a), Maeder (2009), Pinsonneault (2010), Bouvier (2013), Palacios (2013), Mathis (2013), and Montalbán \& Noels (2013).

Early stages to TAMS: For a typical dense molecular cloud, the specific AM is $j_{\text {cloud }} \sim$

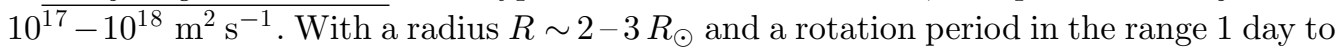
20 days, a typical T Tauri star (age $\sim 10$ Myr) has a specific angular momentum $\sim 10^{12}-$ $10^{13} \mathrm{~m}^{2} \mathrm{~s}^{-1}$ (assuming rigid rotation), a decrease of 5 orders of magnitude. The AM loss occurs through complex hydromagnetic star-disk interactions (Ferreira 2013). Early on the PMS (at a few Myr), the temperature increase due to contraction in the center of the star causes the appearance of a radiative core, some core-envelope decoupling, and the development of differential rotation with radius.

Gallet \& Bouvier (2013) showed the evolution of the surface rotation of stars in several open clusters with ages running from early PMS to mid-MS. The velocity dispersion observed for low-mass stars on the PMS is maintained on the ZAMS $\left(\sim 10 \mathrm{~km} \mathrm{~s}^{-1}\right.$ up to $150-200 \mathrm{~km} \mathrm{~s}^{-1}$ ). This can be explained by the coupling between the magnetized surface of the star and its surrounding disk in the early phase of PMS. This interaction acts as a braking torque on the surface of the star which is forced to rotate at the constant disk rotation rate (disk-locking) as long as the disk does not dissipate, and the disk rotation is taken as the initial condition for the AM evolution of stars when including the PMS evolution. When the disk disappears (at age 5 to $10 \mathrm{Myr}$ ), the gravitational contraction of the star leads to an increase of the uniform rotation rate. Once on the ZAMS (roughly 25-100 Myr), the star evolution slows down and angular momentum can be carried away efficiently by magnetized winds. The spin-down timescale is of order of a few tenths of a Gyr, shorter than the nuclear evolutionary timescale. Between $100 \mathrm{Myr}$ and 1-2 Gyr, the rotation depends on the parameters of the disk-locking description and the description of the magnetized wind AM loss. Later, the rotation follows the Skumanich (1972) spin-down law when surface magnetic braking is operating.

At arrival on the ZAMS, the star does not rotate as a solid body, although this is often taken as an initial condition. Using a simplified two-zone model, Gallet \& Bouvier (2013) suggest that the slow rotators develop a high degree of differential rotation between the radiative core and the convective envelope whereas the faster ones are nearly in solidbody rotation. This is also found when assuming AM transport by MCST (see Marques et al. 2013, Palacios 2013 and references therein). MCST and surface magnetic braking are not able to enforce rigid rotation on the MS (Pinsonneault et al. 1989, Matias \& Zahn 1998) and the core still rotates faster than the surface. On the other hand, AM transport by IGW has been shown to be very efficient in flattening the rotation profile in radiative zones of solar -type stars at the age of the Sun (Charbonnel \& Talon 2005). 
Direct seismic probes of the internal rotation profile of low-mass main sequence stars are not currently possible. Apart from rotation information from open clusters, what we have at our disposal are surface rotation periods for thousands of stars from high quality photometric light curves provided by Kepler's and CoRoT's observations (for instance Affer et al. 2012, McQuillan et al. 2013).

The variations of surface $\mathrm{Li}$ abundance and rotation with age can serve to constrain the AM internal transport (Pinsonneault 1989, Talon 2008b). The surface lithium abundance is too depleted for stars on the MS when using standard models with no transport processes other than atomic diffusion and convection. On the other hand, rotationallyinduced mixing accounts for the observed destruction of $\mathrm{Li}$ on the blue edge of the $\mathrm{Li}$ gap. However, the core-envelope coupling of the MCST approach generates a too-large $\mathrm{Li}$ depletion for stars on the cool side of the $\mathrm{Li} \operatorname{dip}\left(T_{\text {eff }}<6700 \mathrm{~K}\right)$. The AM transport by IGW, by reducing the meridional circulation, limits the mixing, and hence the Li destruction. IGW transport is efficient for stars with a deep enough outer convective region. It can account very well for the cool side of the Li dip.

It would be desirable to have a few stars that, like the Sun, can serve as calibrators for the various prescriptions used to describe the AM surface loss and transport. CoRoT (Michel et al. 2008) and Kepler (Gilliland 2011) provided high-quality photometric data which resulted in very precise high radial order p-mode oscillation frequencies for a large number of low-mass main sequence stars. From the measurement of individual frequencies and their spacings, and careful stellar modelling, the mass and age of a star can be precisely derived. Together with a precise determination of the surface rotation period, such a star can then be used to calibrate the rotation period-age empirical relation, as has been done for the Sun, and to constrain magnetic braking processes for early MS stars. An example is the star HD 52265 which has been observed with CoRoT. The rotation period was also derived to be $P_{\text {rot }}=12.3 \pm 0.15$ days from the CoRoT light-curve variation (Ballot et al. 2011). Using seismic constraints (Roxburgh 2005 and references therein), Lebreton \& Goupil (in preparation) found a precise age of $2.35 \pm 0.25$ Gyr for a mass in the range $1.22-1.27 M_{\odot}$.

Figure 1 displays the surface rotation rate, $\Omega$, as a function of age for HD 52265 and for three types of evolutionary models which correspond to different assumptions about the magnetic braking and AM internal transport prescriptions. The models are computed with the CESTAM code (Marques et al. 2013). The solar value of the magnetic braking constant is obtained for a calibrated solar model with a surface rotation period of 27 days at the solar age (here $K_{\odot}=6.5 \times 10^{47}$ in cgs units). The solar $K$ value is too large for the models to agree with HD 52265 observed values. The seismic constraints impose a very narrow range of possible values for $K \sim(4.06-4.85) \times 10^{47}$ (cgs) as shown in the inset of Fig. 1. Other parameters such as disk lifetime and period have also some influence (not shown here). The result depends on the physical description of the stellar models, in particular the description of AM transport. For instance, the solar value for $K$ becomes acceptable if one assumes an additional viscosity, $\nu_{\text {add }}$, to the canonical prescription for $\nu_{v}$ of the MCST approach (see Sect. 2). The additional viscosity can be seen as mimicking the diffusive transport due to a fossil magnetic field for instance. The adopted value here $\nu_{\text {add }}=3 \times 10^{4} \mathrm{~cm}^{2} \mathrm{~s}^{-1}$ is similar to that found by Decressin et al. (2009) for MS stars and Eggenberger et al. (2012) for a red giant star.

Seismic Constraints Beyond the MS: When the star leaves the main sequence, structural changes occur with the contraction of the inner regions and a huge extension of the envelope. If one assumes local conservation of AM, one must expect a rotation profile increasing toward the interior in the radiative region. When rotationally induced mixing in the radiative region is described by the MCST, it is found that the structural 

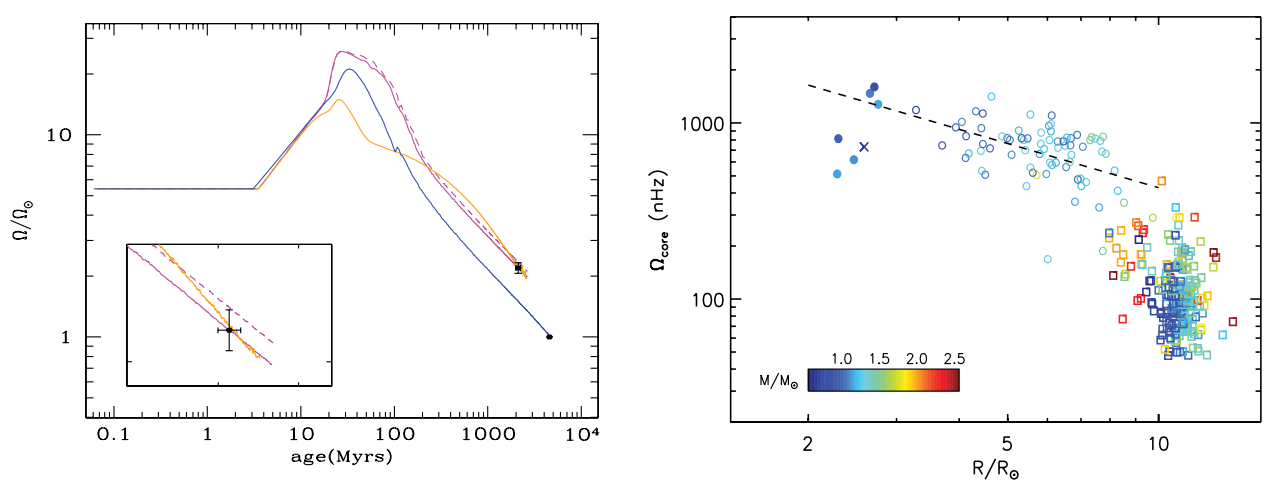

Figure 1. Left: Evolution of the surface rotation normalized to the solar surface rotation versus age for $1.265 M_{\odot}$ models including MCST and for different values of the magnetic braking constant $K$ (solid magenta highest peak, $K=4.54 \times 10^{47}$ (in cgs units), dashed magenta, $\left.K=4.06 \times 10^{47}\right)$. The blue curve (with second-highest peak) represents the evolution for a $1 M_{\odot}$ model with MCST and $K=6.5 \times 10^{47}$ (cgs); the dot indicates the location of the Sun. The cross indicates the position of HD 52265. The orange curve (with the lowest peak) corresponds to $1.265 M_{\odot}$ models with $K_{\odot}$ and additional viscosity (see text). A zoom-in on the location of HD 52265 (black crosses) is shown in the inset. Right: Core rotation rate as a function of the seismic stellar radius for subgiants and red giants. The open symbols correspond to the stars that were studied by Mosser et al. (2012a) (circles: RGB stars, squares: clump stars). The filled symbols correspond to late subgiants and early red giants (Deheuvels et al. 2013) and the cross to a young giant (Deheuvels et al. 2012). (Credit: Deheuvels et al. 2013.)

changes remain dominant over the MCST due to the short evolution timescales (Palacios et al. 2013). Marques et al. (2013) show the evolution of the central and surface rotations for a $1.3 M_{\odot}$ model computed according to MCST from the PMS to the RGB: beyond the TAMS, the surface rotation decreases while the central rotation sharply increases. A steep rotation gradient at the edge of the H-burning shell develops due to rapid core contraction at the TAMS (see also Palacios et al. 2006, Eggenberger et al. 2012). While the surface rotation decreases to $\sim 0.1-1 \mu \mathrm{Hz}$, the central rotation reaches $100 \mu \mathrm{Hz}$ for the subgiant phase and $200 \mu \mathrm{Hz}$ for the RG phase (Marques et al. 2013).

Before 2006, observational constraints on the internal AM transport of low-mass RG stars were coming from the observed surface abundance anomalies. The red giants have long been known to be pulsating variables. However, it is only in the early 2000s that lower mass RG were suspected to oscillate with solar-like oscillations because of their outer convective region. Nonradial solar-like oscillations for red giants were confirmed by the CoRoT (De Ridder et al. 2009) and later Kepler (Bedding et al. 2010).

Subgiants and red giants oscillate with mixed modes (Bedding et al. 2010, Mosser et al. 2011, 2012a). Mixed modes are trapped in two resonant cavities: they behave as gravity modes in the deep interior where both the Brünt-Väisälä and Lamb frequencies are high. In the outer part the modes are trapped as p modes. In between the modes are evanescent (Unno et al. 1989, Dziembowski et al. 2001). In stars where they can be excited, mixed modes can probe the inner structure (for instance the convective cores of $\delta$ Scuti stars (Dziembowski \& Pamyatnykh 1991) or of RG stars (Montalbán et al. 2013). Mixed modes can also serve to probe the inner rotation profile (Goupil et al. 1996).

When the star evolves off the MS, its change of structure modifies the $\mathrm{p}$ and $\mathrm{g}$ cavities and therefore modifies the respective number of $\mathrm{p}$ - and g-dominated modes in the observed frequency spectrum (Montalbán et al. 2010). Observed mixed modes of subgiants are not fully dominated by their g nature and therefore remain sensitive to the surface properties. On the other hand, the red giants are oscillating mostly with g-dominated 
mixed modes which probe the core. Those properties are responsible for specific frequency patterns in subgiant and RG power spectra.

Seismic information provides mass and radius as for MS stars. In addition, due to the presence of g-dominated modes, one is also able to discriminate between clump stars and giant stars (Bedding et al. 2011, Mosser et al. 2011). This information is extremely valuable to investigate the evolution of the rotation profile and to provide constraints on the dominant AM transport during evolution.

For slowly rotating stars, the information on the rotation profile comes from the rotational splittings, $\delta=\int_{0}^{R} K(r) \Omega(r) d r$, where the rotational kernel $K(r)$ involves the associated eigenfunction for the fluid displacement, and $\Omega(r)$ is the rotation profile at radius $r$ in the star. The (p- or g-dominated) nature of the modes strongly influences the behavior of the rotational splittings as a function of frequency (Goupil et al. 2013). The observed rotational splittings of hundreds of giant stars revealed that the rotation of their cores is 5-10 times larger than the surface rotation (Beck et al. 2012, Mosser et al. 2012b, Deheuvels et al. 2012). For the subgiants we have access to both $\Omega_{\text {core }}$ and $\Omega_{\text {env }}$, the surface rotation averaged over the acoustic cavity which corresponds mostly to the convective envelope (Deheuvels et al. 2013). Figure 1b (from Deheuvels et al. 2013) compiles the seismically measured averaged core rotation of subgiants and red giants as a function of the seismically determined stellar radius of the star. The core of the subgiants appears to spin up with evolution, which would mean that the core contraction prevails over AM losses to the envelope. In contrast, at the bottom of the RGB and up the ascent of the RGB, the core rotation of the red giants clearly decreases with evolution. Some efficient AM transport mechanism must operate during these phases (Mosser et al. 2012b). The averaged core rotation of the clump stars has decreased further by a factor of six compared to that of RG stars. However some care must be taken when interpreting the rotational splittings in terms of spin up or down. Indeed the rotational splittings provide an average rotation on central layers which might rotate differentially in radius.

The rotational splittings indicate a mean core rotation of the order of one $\mu \mathrm{Hz}$ to be compared to the $\sim 100-200 \mu \mathrm{Hz}$ typical for subgiant and red giant stellar models at the bottom of the ascending RGB, when assuming AM transport according to MCST. The theoretical rotational splittings are then larger than the observed ones by two orders of magnitude (Eggenberger et al. 2012, Marques et al. 2013, Ceillier et al. 2013).

What could be the origin of the discrepancy? Varying several parameters entering the physical description of stellar models or the chemical composition can decrease the core rotation rate by at most one order of magnitude (Ceillier et al. 2013), the most efficient one being an increase of the horizontal turbulent diffusion coefficient by two orders of magnitude (Marques et al. 2013). In order to mimic an additional AM transport in the stellar models, Eggenberger et al. (2012) add an ad hoc viscosity (representing a yet unknown braking mechanism) in the diffusion coefficient for the AM transport. They found that in order to reproduce the observations for one red giant studied by Beck et al. (2012), the value of the additional viscosity is similar to the value required to account for the observed spin-down of MS slowly rotating solar like stars (Denissenkov et al. 2010). In an attempt to identify some properties of the missing AM transport processes, Ceillier et al. (2013) showed that imposing solid-body rotation on the main sequence only and MCST on the subgiant phase is able to decrease the core rotation by one order of magnitude; again this is not enough to reproduce the observed rotation profiles. The process must also be efficient during the short evolutionary subgiant phase. Two possible missing AM transport processes have so far been identified: a fossil 
magnetic field associated with hydromagnetic instabilities and internal gravity waves. Both have been shown to be efficient in reducing the sharp rotation gradient in stellar models.

\section{Conclusions}

We have briefly reviewed our current understanding of internal AM transport in lowmass stars based on recently collected high-quality data and concomitant theoretical developments. The main result is that the cores of red giants rotate much more slowly than expected. This is in agreement with the seismic results for hot white dwarfs which are found to rotate slowly and must then have lost their AM before the white-dwarf phase (Charpinet et al. 2009). This nevertheless highlights the need for including additional AM transport processes in current stellar models. Many open questions remain. On the theoretical side, more work is needed to model better the impact of the interaction of the star with its circumstellar environment, the impact of instabilities on AM transport, the prescriptions for dynamical effects, the quantitative importance of magnetic fields and IGW. On the observational side, the goals should be the detection of solar-like oscillations for PMS stars, the detection of seismic individual splittings for MS stars and early postMS subgiants. One must then go beyond CoRoT and Kepler results and support the PLATO project (Rauer et al., submitted).

\section{References}

Affer, L., Micela, G., Favata, F., \& Flaccomio, E. 2012, MNRAS, 424, 11

Alvan, L., Mathis, S., \& Decressin, T. 2013, A\&SA, 553, A86

Beck, P. G., Montalbán, J., Kallinger, T., et al. 2012, Nature, 481, 55

Ballot, J., Gizon, L., Samadi, R., et al. 2011, A\&A, 530, 97

Bedding, T. R., Huber, D., Stello, D., et al. 2010, ApJ, 713, 176

Bedding, T. R., Mosser, B., Huber, D., et al., 2011, Nature, 471, 608

Bouvier, J. 2013, EAS Publ. Series, 62, 143

Brun, A. S. \& Palacios, A. 2009, ApJ, 702, 1078

Brun, A. S. \& Rempel, M. 2009, Space Sci. Revs, 144, 151

Ceillier, T., Eggenberger, P., García, R. A., \& Mathis, S. 2013, A\&A, 555, A54

Charbonnel, C. \& Talon, S. 2005, Science, 309, 2189

Charpinet, S., Fontaine, G., \& Brassard, P. 2009, Nature, 461, 501

Decressin, T., Mathis, S., Palacios, A., Siess, L., Talon, S., Charbonnel, C., \& Zahn, J.-P. 2009, $A \& A, 495,271$

Denissenkov, P. A., Pinsonneault, M., Terndrup, D. M., \& Newsham, G. 2010, ApJ, 716, 1269

De Ridder, J., Barban, C., Baudin, F., et al. 2009, Nature, 459, 398

Deheuvels, S., García, R. A., Chaplin, W. J., et al. 2012, ApJ, 756, 19

Deheuvels, S., et al. 2013, ApJ, submitted

Dziembowski, W. A. \& Pamyatnykh, A. A. 1991, A\&A, 248, L11

Dziembowski, W. A., Gough, D. O., Houdek, G., \& Sienkiewicz, R. 2001, MNRAS, 328, 601

Eggenberger, P., Montalbán, J., \& Miglio, A. 2012, A\&\&A, 544, L4

Endal, A. S. \& Sofia, S. 1978, ApJ, 220, 279

Ferreira, J. 2013, EAS Publ. Series, 62, 169

Garaud, P., Meru, F., Galvagni, M., \& Olczak, C. 2013, ApJ, 764, 146

Gallet, F. \& Bouvier, J. 2013, A\&A, 556, A36

Gilliland, R. L. 2011, ASP-CS, 448, 167

Goupil, M.-J., Dziembowski, W. A., Goode, P. R., \& Michel, E. 1996, A\&A, 305, 487

Goupil, M.-J., Mosser, B., Marques, J. P., et al. 2013, A\&SA, 549, A75

Kawaler, S. D. 1988, ApJ, 333, 236

Lecoanet, D. \& Quataert, E. 2013, MNRAS, 430, 2363 
Maeder, A. 2009, Physics, Formation and Evolution of Rotating Stars, Astronomy and Astrophysics Library (Berlin, Heidelberg: Springer)

Maeder, A. \& Zahn, J.-P. 1998, A\&A, 334, 1000

Maeder, A., Meynet, G., Lagarde, N., \& Charbonnel, C. 2013, A\&A, 553, A1

Marques, J. P. \& Goupil, M. J. 2013, in: M. J. Goupil, K. Belkacem, C. Neiner, F. Lignières, \& J. J. Green (eds.), Studying Stellar Rotation and Convection, Lecture Notes in Physics, 865,75

Marques, J. P., Goupil, M. J., Lebreton, Y., et al. 2013, A\&A, 549, A74

Mathis, S. 2011, in: J.-P. Rozelot, \& C. Neiner (eds.), The Pulsations of the Sun and the Stars, Lecture Notes in Physics, 832, 275

Mathis, S. 2013, in: M. J. Goupil, K. Belkacem, C. Neiner, F. Lignières, \& J. J. Green (eds.), Studying Stellar Rotation and Convection, Lecture Notes in Physics, 865, 23

Mathis, S. \& Zahn, J.-P. 2004, AESA, 425, 229

Matias, J. \& Zahn, J.-P. 1998, in: J. Provost, \& F.-X. Schmieder (eds.), Sounding solar and stellar interiors, Proc. IAU Symposium No. 181 (Kluwer Academic), poster volume

Matt, S. P., MacGregor, K. B., Pinsonneault, M. H., \& Greene, T. P. 2012, ApJ, 754, L26

McQuillan, A., Aigrain, S., \& Mazeh, T. 2013, MNRAS, 432, 1203

Meynet, G., Ekström, S., Maeder, A., et al. 2013, in: M. J. Goupil, K. Belkacem, C. Neiner, F. Lignières, \& J. J. Green (eds.), Studying Stellar Rotation and Convection, Lecture Notes in Physics, 865, 3

Mestel, L. 1953, MNRAS, 113, 716

Michel, E., Baglin, A., Auvergne, M., et al. 2008, Science, 322, 558

Montalbán, J. \& Noels, A. 2013, in: J. Montalbán, A. Noels, \& V. Van Grootel (eds.), Ageing Low Mass Stars: From Red Giants to White Dwarfs, European Physical Journal Web of Conferences, 43, id. 03002

Montalbán, J., Miglio, A., Noels, A., Scuflaire, R., \& Ventura, P. 2010, ApJ, 721, L182

Montalbán, J., Miglio, A., Noels, A., Dupret, M.-A., Scuflaire, R., \& Ventura, P. 2013, ApJ, 766,118

Mosser, B., Barban, C., Montalbán, J., et al. 2011, A\&A, 532, A86

Mosser, B., Goupil, M. J., Belkacem, K., et al. 2012a, A\&A, 540, A143

Mosser, B., Goupil, M. J., Belkacem, K., et al. 2012b, A\&AA, 548, A10

Palacios, A. 2013, EAS Publ. Series, 62, 227

Palacios, A., Charbonnel, C., Talon, S., \& Siess, L. 2006, A\&A, 453, 261

Paxton, B., Cantiello, M., Arras, P., et al. 2013, ApJS, 208, 4

Pinsonneault, M. 1997, ARAA, 35, 557

Pinsonneault, M. H. 2010, in: C. Charbonnel, M. Tosi, \& F. Primas (eds.), Light Elements in the Universe, Proc. IAU Symposium No. 268 (Cambridge, UK: Cambridge University Press), p. 375

Pinsonneault, M. H., Kawaler, S. D., Sofia, S., \& Demarque, P. 1989, ApJ, 338, 424

Potter, A. T. 2012, Ph.D. Thesis, University of Cambridge

Press, W. H. 1981, ApJ, 245, 286

Reiners, A. \& Mohanty, S. 2012, ApJ, 746, 43

Rogers, T. M., Glatzmaier, G. A., \& Jones, C. A. 2006, ApJ, 653, 765

Roxburgh, I. W. 2005, A\&A A, 434, 665

Schatzman, E. 1993, A\&SA, 279, 431

Schatzman, E. 1962, Annales d'Astrophysique, 25, 18

Skumanich, A. 1972, ApJ, 171, 565

Strugarek, A., Brun, A. S., \& Zahn, J.-P. 2011, A\&A, 532, A34

Talon, S. 2008a, EAS Publ. Series 32, 8

Talon, S. 2008b, MemSAIt, 79, 569

Unno, W., Osaki, Y., Ando, H., Saio, H., \& Shibahashi, H. 1989, Nonradial oscillations of stars (Tokyo: University of Tokyo Press)

Zahn, J.-P. 1992, A\&A, 265, 115

Zahn, J.-P. 2007, EAS Publ. Series, 26, 147

Zahn, J.-P. 2009, CoAst, 158, 27 